\title{
Optimization and Matching Scheme of Public Management Resources for Industry 4.0 and Smart City
}

\author{
Jinglin Fan (D) and Lei Shi \\ School of Architecture \& Art, Central South University, Changsha 410075, China \\ Correspondence should be addressed to Jinglin Fan; jinglin2021@csu.edu.cn
}

Received 15 August 2021; Accepted 6 September 2021; Published 12 October 2021

Academic Editor: Shaohui Wang

Copyright (C) 2021 Jinglin Fan and Lei Shi. This is an open access article distributed under the Creative Commons Attribution License, which permits unrestricted use, distribution, and reproduction in any medium, provided the original work is properly cited.

\begin{abstract}
With the development of Big Data, Industry 4.0, and other technologies, the concept of smart city has become a new goal, new concept, and new practice of many urban developments. It provides a method to solve the problem that public management cannot optimize resources in China's urban development and puts forward a supporting scheme more in line with the optimization of public management resources. Effective use of relevant supporting schemes can improve urban public management capacity, optimize resources, and promote the city to embark on the road of scientific development. This paper starts with the multiobjective optimization algorithm to optimize the matching of public resources and realize the effective utilization of public management resources. Using particle swarm optimization algorithm, the optimal allocation management of 8 kinds of resources in this paper is carried out, and the optimization analysis is carried out from the performance indexes, such as resource allocation time and configuration complexity. Finally, the weights of the eight resources in importance, complexity, and resource demand are $0.4,0.4$, and 0.2 , respectively. The proposed method realizes the classification of resources and the optimal matching of resources.
\end{abstract}

\section{Introduction}

Driven by the tide of urban informationization and the rise of data science, smart cities have become a new concept and practice of future urban development on a global scale. Data management, application, and analysis technologies, such as Big Data, data activation, and data mining, play a central role in the construction of smart cities [1]. At present, all local governments in China are actively carrying out the planning and construction of smart cities. Smart cities provide a means to solve the problems faced by China's urban development [2]. To optimize and maximize the use of limited resources, attention should be paid to the rational distribution of resources. As reasonable and effective allocation of resources can promote the development of cities, random and arbitrary allocation of resources may hinder the development of cities. Resources are limited in time and space, and missing is an error. Matching is a powerful statistical tool in design and analysis [3]. Optimal allocation of resources makes the team dynamic, efficient, flexible, and competitive [4]. To regard people as the most valuable asset, all performance and so on are based on this as a prerequisite. Researchers put forward that the innovation of public sector involves computerization, continuous optimization, adapting to the changes, and making its image more suitable for the needs of smart city construction. Arbolino et al. [5] proposed a new method for selecting public administrationfunded projects, which is suitable for the planning of sustainable tourism activities and can maximize the allocation efficiency. In addition, we have added the consideration of environmental, social, and economic impacts to the multiobjective optimization model to achieve maximum utility. Hergüner [6] verified exploratory factor analysis (EFA) and confirmatory factor analysis (CFA) in the form of questionnaires and proposed a CC evaluation model. The model consists of five key attributes, which involve all aspects of cultural ability. It can not only evaluate the CC level of students majoring in public administration but also evaluate the responsiveness of public institutions to the public. Liu et al. [7] put forward the optimal allocation method of public 
resources based on genetic algorithm and cloud computing, which takes the running time and cost of public resources as the optimization objectives, and put forward the adaptive function of population constraint. Using individuals in genetic algorithm to optimize the objectives of genetic algorithm and complete the optimal allocation of related public resources, the method can improve the efficiency of resource operation and has high reliability. The multiobjective proposed in this paper is to optimize the resource matching of smart cities, which needs to optimize the human resources, public resources, and information resources in cities. The objectives refer to the target requirements such as time, task coupling, and resource demand. By optimizing these goals, we can optimize the resources of smart cities and maximize the benefits.

\section{Introduction of Related Theoretical Basis}

2.1. Brief Introduction of Smart City. Smart city originates from the media field, which refers to the open integration of urban systems and services using various information technologies or innovative ideas, so as to improve the efficiency of resource utilization, optimize urban management and services, and improve the quality of life of citizens. It is an advanced urban computerization form that fully applies the new generation of information technology to all fields of urban life on the basis of the new generation innovation in the knowledge society [8].

Since IBM CEO Peng Mingsheng first put forward the concept of "Smart Earth" in 2009, the construction of "Smart City" is in full swing all over the world, and thousands of cities around the world have started the "Smart City" project [9]. Hundreds of cities and regions in China have successively built "smart cities."

Figure 1 shows the general construction of Big Data platform in smart city construction.

\subsection{Brief Introduction of Public Management Resources.} Public management resources are common goods held by people in specific communities, that is, public property, which is used for public services. As the content of public administration, it has specific significance and scope, that is, tangible and intangible property shared by residents in specific communities. These resources are named after possessions that everyone can enjoy, but actually no one can make full use of them. These resources mainly include public information and natural resources.

\subsubsection{Brief Introduction of Public Resource Allocation Theory.} Resource allocation is understood as the allocation of insufficient resources after matching them according to their functions. Resource allocation means that society allocates its available resources to different uses within a certain range. The characteristics of common resources make it necessary to attach further matching before it can face the market. Recently, with the continuous development of socialism and the continuous improvement of market economy, the reform of matching public resources in China has been further

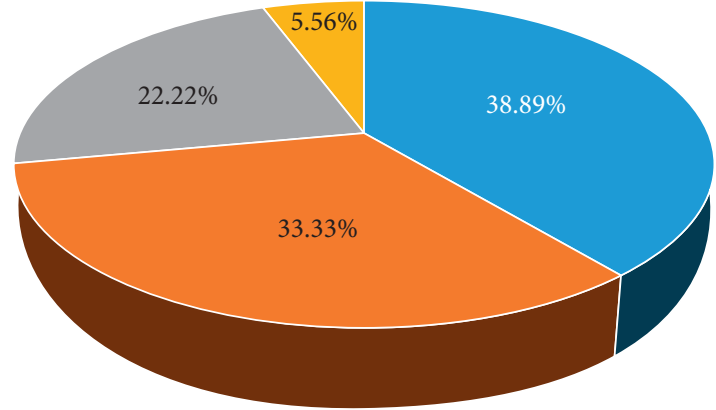

- A government affairs platform is being built

- A government affairs platform has been built

- City-level platform is under construction

- City-level platform has been built

Figure 1: Construction of urban Big Data platform.

promoted, and public resources have been better utilized and improved. The power of administrative organs has been strengthened, social fairness and justice have been further strengthened, and significant progress has been made in anticorruption construction. Public resources are competitive, but this does not mean that their influence on others and affairs is single. The continuous marketization of public resources optimization and matching shall not violate relevant market rules. Under the regulation and control of relevant state departments, prices are adjusted according to their own scientific systems and steps, and the distribution of public resources is controlled in the form of market competition. Recently, the practical experience in some areas shows that the benefits brought by further improving the matching of public resources are particularly obvious [10].

\subsection{Brief Introduction of Multiobjective Optimization.} Multiobjective optimization problem is an optimization problem involving multiple optimization objectives. In an optimization problem, if there is only one optimization objective, then the problem is a single-objective optimization problem. For example, "the fastest task is completed," in which, there is only one optimization goal-"minimizing time." However, in actual scenarios, many instances need to be optimized for more than one goal. For example, "maximizing income and minimizing time" exist at the same time, and there is a conflict between them, that is, "maximizing income" will lead to the increase of consuming "time," and vice versa. Then, this kind of problem belongs to MOP [11].

Generally, MOP is a minimization problem, and its general form can be expressed as

$$
\begin{aligned}
\operatorname{minimize} F(\mathrm{x}) & =\left(F_{1}(x), F_{2}(x), \ldots, F_{M}(x)\right) \\
\text { subject } x & \in \Omega .
\end{aligned}
$$

where $x$ represents the solution vector in the feasible solution space $\Omega . F_{i}(i=1,2, \ldots, M)$ is the $i$ th goal that needs to be minimized. In particular, if you want to maximize an objective function, you can minimize its negative value to achieve the same optimization effect. 
In MOP, there is generally no feasible solution to minimize all objective functions at the same time, so the concept of Pareto dominance is introduced. PD is defined as follows.

If $x, y \in \Omega$ and $x<y$, then it is equivalent to

$$
\begin{aligned}
\forall_{i} \in(1,2, \ldots, M): & F_{i}(x) \leq F_{i}(y) \wedge \exists_{j} \in(1,2, \ldots, M) \\
: & F_{j}(x) \leq F_{j}(y),
\end{aligned}
$$

where $x<y$ means $x$ dominates $y$. In addition, the decision vector that is not dominated by any other vector is Pareto optimal. The optimal solution set in decision space is Pareto solution set, and the corresponding set of its corresponding target vector is Pareto frontier.

Multiobjective optimization algorithms have been developed up to now, among which the classical algorithms are nondominated sorting genetic algorithms with elite strategy [12] and multiobjective evolutionary algorithms based on decomposition [13]. As far as the current research is concerned, the commonly used multiobjective optimization algorithms include multiobjective optimization based on artificial immune system and so on.

In recent years, with the deepening of research, researchers have gradually applied various technologies, such as biology, physics, artificial intelligence, and computer science, to the field of optimization algorithms and proposed various intelligent optimization algorithms to solve scheduling problems. Owing to the advantages of wide application, high efficiency, and easy to obtain satisfactory solutions, intelligent algorithms are often used to solve matching optimization problems.

\subsubsection{Brief Introduction of Standard Particle Swarm Opti-} mization Algorithm. Particle swarm optimization (PSO) is an evolutionary algorithm based on swarm intelligence algorithm. It operates individual particles according to the value of objective function. Different from other optimization algorithms, particle swarm optimization algorithm regards each individual as a particle whose volume and mass are ignored when flying at a certain speed in $D$-dimensional solution space. In the iteration process, the particle has velocity vector $v_{i d}$ and position vector $s_{i d}$ to describe its state.

The specific implementation steps are as follows:

Step 1: select the population size and initialize the speed and position of particles in the population; it is generally defined that $C 1=C 2=1.5$, with population size $m$, evolution times equal to 1000, random position Pos, and velocity $V$.

Step 2: calculate the fitness value of each particle.

Step 3: update the current optimal position $P_{i}^{b}$ of particles, compare the fitness value calculated in Step 2 with the previous optimal position of each particle; if it is better, select the current moderate value as $P_{i}^{b}$; otherwise, retain the original value.

Step 4: update the global optimal position $p g^{b}$, compare the moderate value calculated in Step 2 with the current global optimal position for each particle, and select the current moderate value as $p g^{b}$ if it is better; otherwise, retain the original value.

Step 5: update particle velocity and position.

Step 6: determine whether to end the search, if the condition is met (the error is good enough or the maximum number of cycles is reached); otherwise, return to Step 2.

The flow chart of the basic particle swarm optimization algorithm is shown in Figure 2:

\section{Optimization and Matching Analysis of Public Management Resources Based on Smart City}

\subsection{Classification of Public Management Resources.}

(1) Public facilities: they are public goods. All people in a specific community can enjoy and benefit from material existence, that is, urban streets and bridges. Specific communities benefit from it, which is only a public product that some people can benefit from, but it does not belong to the field of public management. For small subordinate public entities, it should be public property, but for small subordinate public entities, public property is not necessarily public property for superior public entities, but "public goods."

Public property refers to public facilities and public goods that all people in specific communities can enjoy and benefit from. It is different from natural resources in that public goods must first be a raw product. It does not mean that all members of a certain community can use it, but only public goods that can be used by some people, which do not belong to the scope of public management. For example, roads, bridges, and parks are public goods, and restricted items do not belong to the scope of public management.

(2) Public information resources: people in specific communities share and may own various spiritual products, including scientific and technological achievements and economic information. Among them, the basis of development and survival is generally material, information, and energy. In modern human society, information is an extremely important resource and occupies a major position. There are also two types of information resources: one is the information resources that people in a specific community can own and enjoy and the other is the information that people in a specific range can use. Only the first one is the object of public administration.

Public information refers to various spiritual products that all members of a specific community can own and enjoy, such as economic information, scientific and technological achievements, and 


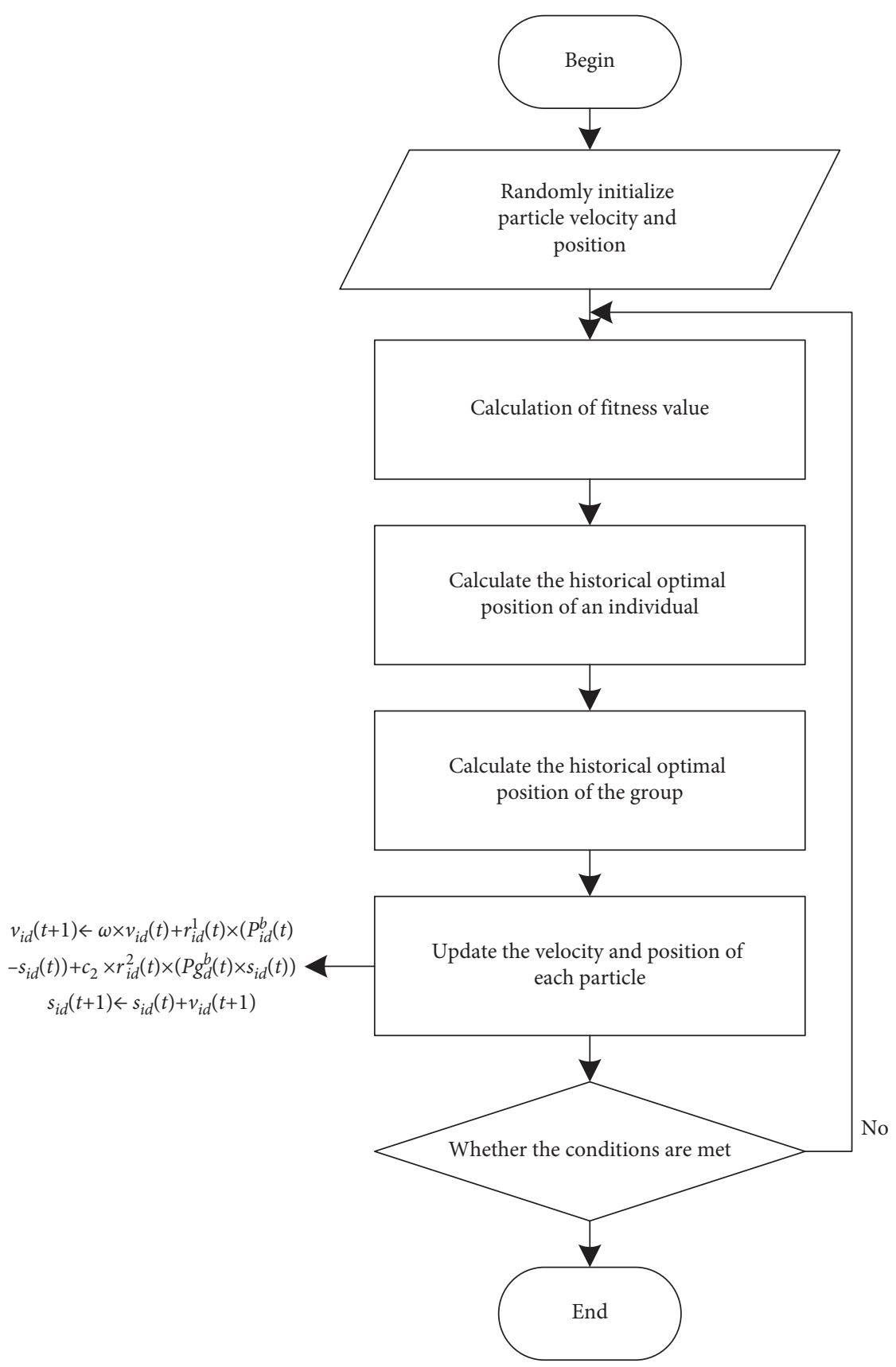

FIgURE 2: Flow chart of basic particle swarm optimization algorithm.

cultural products. Information is now playing an increasingly important role. Information in public administration is about information shared and available from members of a particular community, rather than information held and enjoyed by certain people.

(3) Public human resources: they are social resources created by work and talents. Human resources are the most precious and active among all kinds of social resources. Therefore, its use and development has become a problem that some public administrative departments need to solve. Human resource management includes training (training, motivation, renewal, and orientation) and the use and development of human resources. It does not include the management, development, training, and use of internal talents by public administration institutions. Only the management of human resources shared by the community belongs to the scope of public administration.

(4) Public natural resources: the conditions and foundations of various natural materials on which a specific society depends for development and survival, such as mineral resources, land resources, water resources, and forest resources. Obviously, these are public property. As a specific community, 
they need to rationally use and develop it. Protection and reconstruction have a great impact on the overall development of society. Therefore, it should become an important part of public management.

(5) Public enterprises and companies: they are public enterprises established with state investment. Public enterprises are jointly owned by citizens. They are created by taxpayers' income and public investment. Therefore, it is the responsibility of the public administration to manage them well. Among them, the scale of public enterprises managed by different types of public institutions is different. One of the fundamental tasks of public administration in managing public enterprises is to maintain and increase value and create public interests, which is the purpose of public investment. As a socialist country, China has abundant real estate resources of state-owned and state-owned enterprises.

Therefore, resources are divided into two categories: social resources and natural resources. Natural resources are equal to the public natural resources in this paper. Social resources are classified into (1) public enterprises and companies, (2) public information resources, (3) Public facilities and public goods, and (4) public human resources. The classification of common management resources is shown in Figure 3.

\subsection{Matching of Public Management Resources in Smart} Cities. The concept of public management is relatively backward. In public service and social management, the government management mode lags behind. In public administration, "senior managers" have greater power. This concept facilitates the management of a service to a certain extent, but it cannot fundamentally meet the needs of the people. At the same time, the degree of cooperation between different departments in smart cities is low, so they must obey the system deployment, reflect the characteristics of service-oriented government, and adapt to the characteristics of cooperation and implementation of networked smart cities. Backward ideas are not only detrimental to government public administration but also affect the innovation of public administration because the existence of old ideas cannot fully realize the value of smart city and will also affect the efficiency of public management.

The mechanism of public administration is relatively backward. First of all, the administrative procedures are complicated. To facilitate management and reduce workload, some administrative departments blame people for problems that should be solved by different departments. People's patience reduces their satisfaction with government public administration. Second, administrative agencies are overloaded. In the past, the government managed all aspects of people's social life, and a public administration function was often divided into several departments. After entering the market economy, "administration" gradually changed into "service." The former public administration system could not meet the rapidly changing economic and social needs. With the rapid development of the Internet, it is urgent to straighten out the overburdened public administration department. Third, management functions overlap. The vague definition of the functions of some administrative departments leads to small-scale conflicts and quarrels among various departments when exercising their public administrative functions, which harms the interests of the masses. Public administration within the framework of smart cities must be efficient and transparent; at present, the backward governance mechanism cannot adapt to the new public administration model, which slows down the innovation of public administration.

Infrastructure construction is slow, and the level of application, development, and popularization is low. Although the construction of smart cities in the field of transportation is developing rapidly in various cities, which can report traffic congestion and public transportation in time, the progress in other fields is still slow. On one hand, the operation of smart city system should rely on infrastructure construction. Due to the limitation of technology and resources, the progress of infrastructure construction is still slow. On the other hand, if citizens want to participate in the smart city system, they must install corresponding applications on mobile terminals. However, the current progress of application development is not ideal, and the funds are insufficient. There are still many citizens who do not know how to participate in the smart city system. Without the participation of citizens, accurate information cannot be obtained, and their feedback and needs cannot lead to targeted innovation in public management.

\section{Design and Implementation of Optimal Matching Model of Public Management Resources in Smart City}

\subsection{Based on Resource Classification in Public Resource} Management. According to the five categories of resources in public resource management, to facilitate the calculation of this paper, it is divided into two categories: renewable resources and nonrenewable resources. Among them, nonrenewable resources, that is, public natural resources, can also be referred to as natural resources. Renewable resources can be divided into two categories: public human resources and other renewable resources, where, other renewable resources are classified into public enterprises, companies, public information resources, public facilities, and public goods as shown in Figure 4.

4.1.1. Renewable Resource. Human resource management includes training, use, and development of human resources and many other aspects. Among them, training is divided into training, motivation, renewal, and orientation.

As in the management of public resources, each person performs his or her own duties but is related to each other and assigns tasks and positions according to his or her own experience level, subjective preference, and good skills.

Assuming that the work task in a city can be divided into $n$ subtasks, that is, $T=\left\{T_{1}, \ldots, T_{n}\right\}$, it is necessary to select 


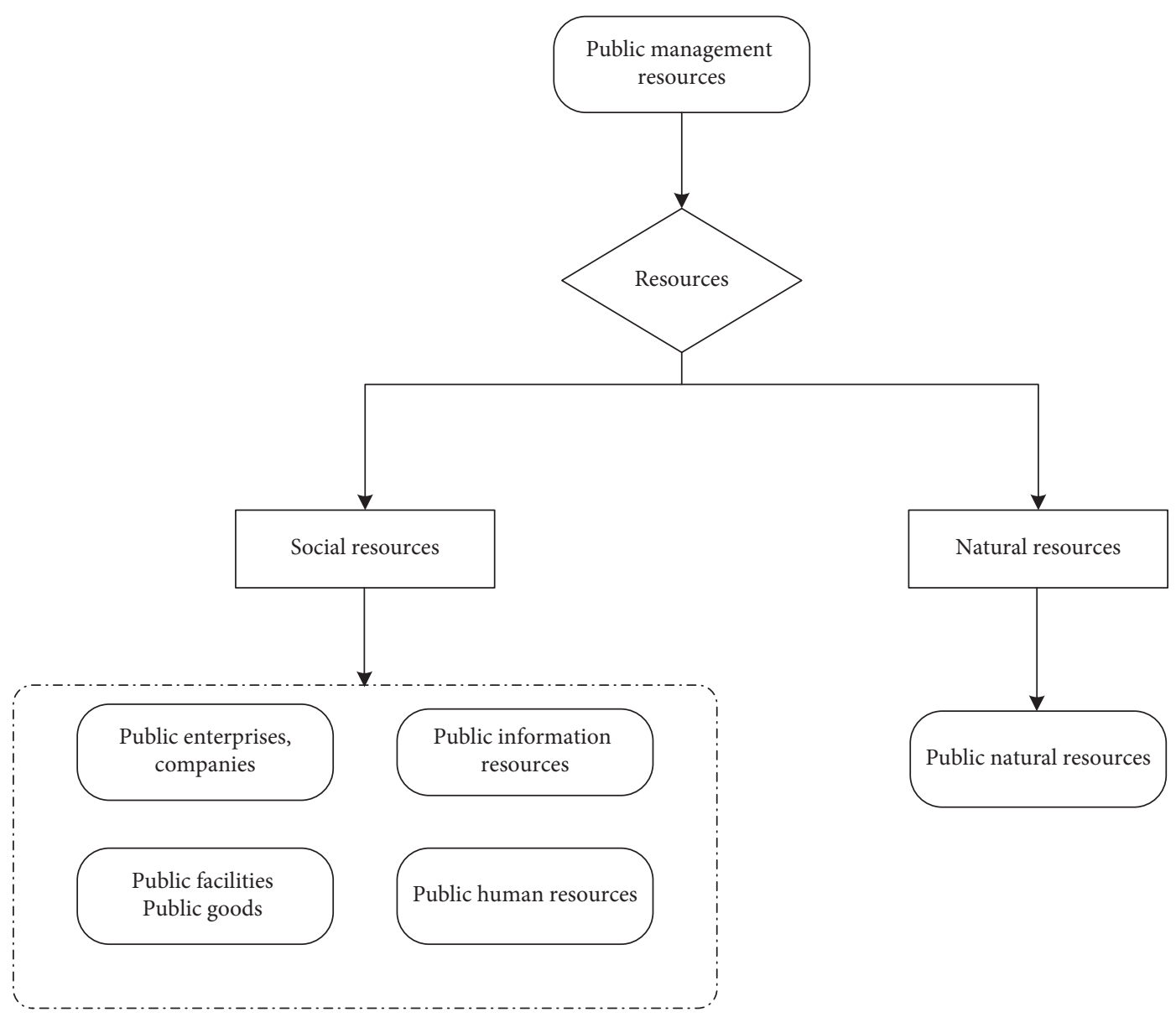

Figure 3: Classification of common management resources.

the appropriate department from $m$ departments to complete the task, and the $m$ departments are set as $O_{1}, \ldots, O_{j}, \ldots, O_{m}$, and according to the task, the personnel combination of the corresponding departments will be selected to form a team to perform the task, and the number of staff in each department is $K_{1}, \ldots, K_{j}, \ldots, K_{m}$, respectively (Figure 5.

As the members of the city's public management team come from different departments, different regions, and have different knowledge backgrounds and social experiences, it is necessary to classify and quantify the abilities of personnel and match them with the abilities required by actual tasks. Specific required capabilities should be classified according to specific tasks.

In the process of matching related tasks and personnel in public resource management, the working ability level of staff in related departments or teams needs to be described qualitatively; this is fuzzy and uncertain. It is necessary to provide scientific data as the basis for matching tasks with personnel, to quantify research from qualitative factors, and to objectively distinguish their objective ability level. Because of the fuzziness of the description of ability level, this paper uses expert fuzzy evaluation method to quantitatively evaluate the ability level of personnel and teams [14]. The utility index of relevant personnel ability characteristics can be divided into five grades: very low (0-0.2), low (0.2-0.4), medium (0.4-0.6), high (0.6-0.8), and very high (0.8-1).

The calculation steps of capability level are as follows:

Step 1: according to the actual situation of the staff, its experts score the relevant ability level of each staff. Suppose $P_{j k}$ is the Kth staff member of the $j$ th department $O_{j}$, and its ability level is defined.

$$
\text { Ability }_{j k}=\left\{A_{j k}^{\mathrm{fir}}, A_{j k}^{\mathrm{sec}}, A_{j k}^{\mathrm{thi}}, \ldots\right\} .
$$

Step 2: match the ability level of the formed team accordingly, and record it as

$$
\text { Ability }_{j}=\left\{\frac{\sum_{k=1}^{k_{j}} A_{j k}^{\mathrm{fir}}}{k_{j}}, \frac{\sum_{k=1}^{k_{j}} A_{j k}^{\mathrm{sec}}}{k_{j}}, \frac{\sum_{k=1}^{k_{j}} A_{j k}^{\mathrm{thi}}}{k_{j}}, \ldots\right\} .
$$

Step 3: according to the priority of the task, the experts score the ability required for the task, which is recorded as

$$
\mathrm{RA}=\left\{\mathrm{RA}^{\mathrm{fir}}, \mathrm{RA}^{\mathrm{sec}}, \mathrm{RA}^{\mathrm{thi}}, \ldots\right\}
$$

Step 4: according to the emphasis of tasks to be executed on various ability levels, the weight value of ability level is 


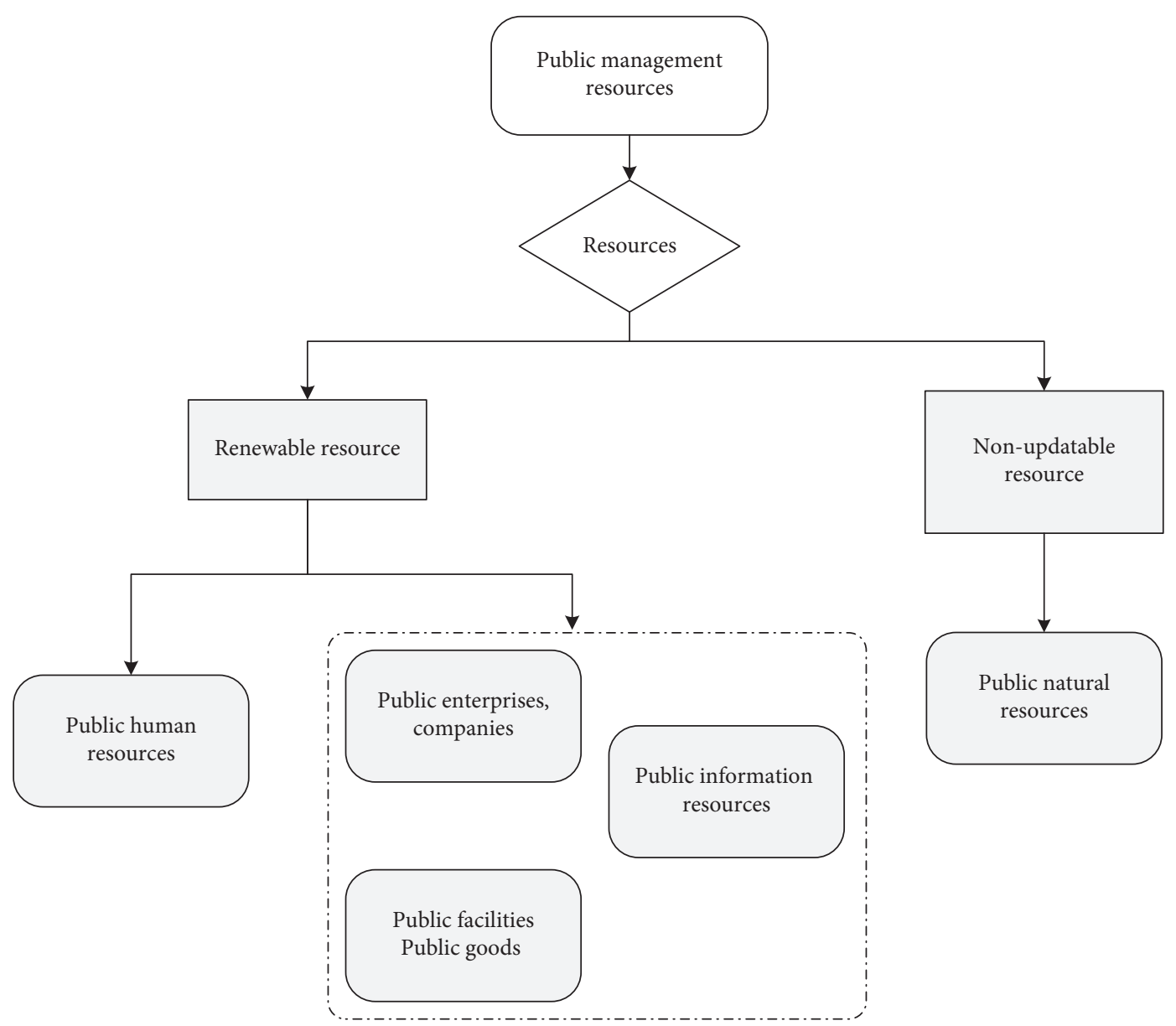

FIgURE 4: Resource classification according to common resource management clock.

obtained using the step fuzzy number complementary judgment matrix method $\eta_{1}, \eta_{2}, \eta_{3}, \ldots, \eta_{n}$.

$$
\eta_{1}+\eta_{2}+\eta_{3}+\cdots+\eta_{n}=1
$$

Step 5: calculate the matching degree between tasks and the abilities of each person:

$A_{i j}^{\text {matching }}=\left\{1 \frac{\sum_{k=1}^{k_{j}} A_{j k}^{\mathrm{fir}}}{k_{j} R A^{f i r}}+\eta_{2} \frac{\sum_{k=1}^{k_{j}} A_{j k}^{\mathrm{sec}}}{k_{j} R A^{\text {sec }}}+\eta_{3} \frac{\sum_{k=1}^{k_{j}} A_{j k}^{\mathrm{thi}}}{k_{j} R A^{\text {thi }}}, \ldots\right\}$.

Personnel preference can be used to describe the motivation and enthusiasm of personnel for tasks. The higher the value, the greater the interest and responsibility of the member for tasks. Pre ${ }_{i j k}$ indicates the preference value of the $k$ th worker in the group of the $j$ th cooperative department for the $i$ th task. The larger the value in $[0,1]$, the higher the preference degree. Personnel preferences are calculated by tasks performed by the cooperation department for different personnel.

The preference set of personnel $P_{j k}$ for tasks is $\operatorname{Pre}_{i j k}=\left\{\operatorname{Pre}_{1 j k}, \operatorname{Pre}_{2 j k}, \ldots, \operatorname{Pre}_{n j k}\right\}$. If the average value of preference values of a team member is taken as the preference value of the team, the preference value of the team is as follows:

$$
\operatorname{Pre}_{j}=\left\{\frac{\sum_{k_{j}}^{k_{j}} \operatorname{Pre}_{1 j k}}{k_{j}}, \frac{\sum_{k_{=1}}^{k_{j}} \operatorname{Pre}_{2 j k}}{k_{j}}, \ldots, \frac{\sum_{k_{=1}}^{k_{j}} \operatorname{Pre}_{n j k}}{k_{j}}\right\} .
$$

The preference matrix for that person is Pre $=\left(\operatorname{Pre}_{i j}\right)_{n \times m}$.

As the urban details of each city are different from the region of the city, the management methods of each city are adapted to local conditions, and the requirements of teams and personnel targeted by each task are different. The comprehensive matching matrix between team and work task is $M=\left(m_{i j}\right)_{n \times m}\left(\gamma^{A}\right.$ and $\gamma^{P}$ are the weight values of ability matching degree and preference value, $\gamma^{A}+\gamma^{P}=1$ ).

$$
m_{i j}=\gamma^{A} A_{i j}^{\text {matching }}+\gamma^{P} \operatorname{Pre}_{i j} .
$$

Suppose that in the task of managing resources in public, other renewable resources are $R^{\text {res }}=\left\{R_{1}^{\text {res }}, R_{1}^{\text {res }}, \ldots, R_{l}^{\text {res }}, \ldots, R_{f}^{\text {res }}\right\}$, where $f$ is the renewable type, $R_{l}^{\text {res }}$ represents the first resource, and $S_{l}^{\text {res }}$ is the available supply of $R_{l}^{\text {res }}$, then the following constraints exist for other renewable resources: $\sum_{i \in I_{t}} S_{i l}^{\text {res }} \leq S_{l}^{\text {res }}, \forall l, t$, where $S_{i l}^{\text {res }}$ represents the supply of other renewable resources $R_{l}^{\text {res }}$ 


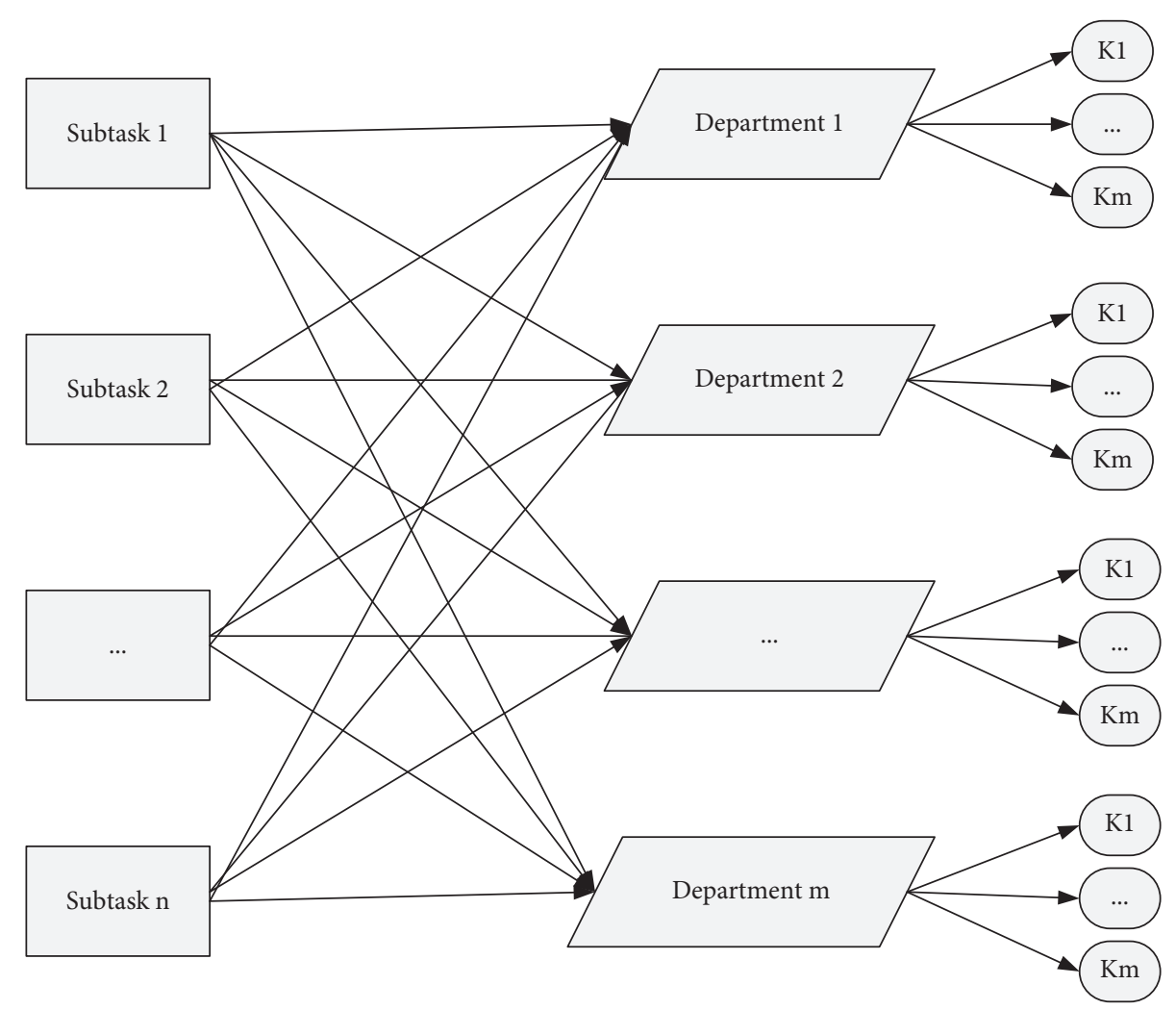

FIGURE 5: Mapping diagram of task and department matching.

to task $T_{i}$ and $I_{t}$ represents the task set in execution state at time $t$.

As there are differences in resource structure and function, their effects on tasks are also different. After the matching resources are given to the task, it can effectively improve the execution efficiency of the task and shorten the actual execution time of the task. Therefore, reasonable matching resources can effectively shorten the working time period. To do this, performance metric $R I^{\text {res }}$ is introduced, which indicates the degree of resources to work tasks [15]. Take the average resource performance index as $R I_{\text {ave }}^{\text {res }}=1$, $R I^{\text {res }}<1$ if the performance level is lower than the average level, and $R I^{\text {res }}>1$ if it is higher than the average level. The higher the resource performance index, the greater the contribution to the completion of work tasks and the greater the corresponding performance index. The performance index of resources is given by expert fuzzy evaluation method.

Owing to the reusability of renewable resources, in the matching process, some resources are in a long-term matching state, while some resources may be idle all the time, which will inevitably lead to waste of resources. To avoid the cost increase caused by idle resources, this paper introduces the utilization rate of renewable resources $U^{\text {res }}$ to measure the overall use of renewable resources, as follows:

$$
\mathrm{UR}^{\mathrm{res}}=\frac{\sum_{i=1}^{n} \sum_{l=1}^{f} s_{i l}^{\mathrm{res}} \mathrm{PT}_{i}}{\sum_{l=1}^{f} s_{l}^{\mathrm{res}} \mathrm{TF}}
$$

where $\mathrm{TF}$ is the completion time of the whole development project, $\mathrm{PT}_{i}$ is the actual execution time of task $i$, and taskresource satisfaction sat ${ }_{i l}^{\text {res }}$ is introduced to express the satisfaction degree of task $T_{i}$ to the allocated updatable resource $R_{l}^{\text {res }}$, as follows:

$$
\text { sat }_{i l}^{\mathrm{res}}=\frac{\mathrm{RI}_{l}^{\mathrm{res}}}{\mathrm{RI}(\text { need })_{i}^{\mathrm{res}}},
$$

where $\mathrm{RI}$ (need) ${ }_{i}^{\text {res }}$ represents the performance requirements of task $T_{i}$ for the updatable resource $T_{i}$.

In this paper, "other resources" refers to the reuse of renewable resources, which makes some resources in a longterm matching state, while some resources may be idle all the time, which will inevitably lead to waste of resources, to avoid the cost increase caused by idle resources. It can be specifically called renewable resource utilization rate to meet the rational allocation of resources and achieve the best balance state.

4.1.2. Nonupdatable resource. The total supply of nonrenewable resources, that is, natural resources, remains unchanged throughout the task, and the sum of natural resources used in each work at any time of the task cannot exceed the total supply of this resource.

Nonrenewable resources belong to consumable resources, with limited total amount and corresponding resource constraints. Therefore, this type of resource should be carefully considered in the process of matching with tasks. That is, tasks with higher priority match 
natural resources first. Suppose the natural resource $R^{n-\text { res }}=\left\{R_{1}^{n-\text { res }}, R_{1}^{n-\text { res }}, \ldots, R_{g}^{n \text {-res }}, \ldots, R_{h}^{n \text {-res }}\right\}$, where $h$ is the renewable resource type, $R_{g}^{n-\text { res }}$ is the gth nonrenewable resource, that is, natural resources. Remember $s_{g}^{n \text {-res }}$ as the available supply of $R_{g}^{n-\text { res}}$; then, there is the following constraint $\sum_{i} s_{i g}^{n-\text { res }} \leq s_{g}^{n-\text { res }}, \forall g$, where $s_{i g}^{n-\text { res }}$ represents the supply of nonrenewable resource $R_{g}^{n-\text { res }}$ required by task $T_{i}$.

Same as mentioned earlier, this article introduces the performance index $\mathrm{RI}^{\mathrm{res}}$, which indicates the degree of natural resources to work tasks. Take the average resource performance index as $R I_{\text {ave }}^{\text {res }}=1, R I^{\text {res }}<1$ if the performance level is lower than the average level, and $R I^{\text {res }}>1$ if it is higher than the average level. The higher the resource performance index, the greater the contribution to the completion of work tasks and the greater the corresponding performance index. The performance index of resources is given by expert fuzzy evaluation method.

In addition, task-resource satisfaction sat ${ }_{i g}^{\text {res }}$ is introduced to indicate the satisfaction degree of task $T_{i}$ with the allocated nonupdatable resource $R_{g}^{n-\text { res }}$, as follows:

$$
\text { sat }_{i g}^{\text {res }}=\frac{R_{g}^{n-\text { res }}}{\mathrm{RI}(\text { need })_{i}^{\text {res }}},
$$

where RI(need) ${ }_{i g}^{\text {res }}$ indicates the performance requirements of task $T_{i}$ for updatable resource $R_{l}^{\text {res }}$

Introduce $U R^{n-\text { res }}$ to measure the overall situation of nonupdatable resources as follows:

$$
\mathrm{UR}^{n-\text { res }}=\frac{\sum_{i=1}^{n} \sum_{g=1}^{f} s_{i l}^{\text {res }} \mathrm{PT}_{i}}{\sum_{g=1}^{f} s_{l}^{n-\text { res }} \mathrm{TF}} .
$$

\subsection{Optimization Model Priority Calculation}

4.2.1. Subtask Priority Judgment. By judging the priority of subtasks in tasks, the matching order of related resources is carried out. The rules for judging priority can be as follows:

(1) Task time, that is, start time, execution time, and so on. The priority rules based on time are as follows: shortest task cycle priority, earliest start time priority, and earliest finish time priority.

(2) Task coupling degree, that is, the critical degree of each subtask in the execution and its influence on the execution of other subtasks or subsequent tasks. The rules based on the degree of task coupling include giving priority to the degree of association of subsequent tasks.

(3) The intensity of task demand for resources, that is, the demand intensity of resources required by each subtask. The priority rules based on resources include priority of the maximum amount of resource demand.

(4) Mixed aspects: based on the aforementioned three points, analyze at the same time and comprehensively consider and judge the priority.
Based on the aforementioned four points, the task priority can be calculated from the following three aspects. It can be expressed by task time and coupling degree.

The importance of subtask $T_{i}$ is calculated as follows: (where $T_{i}^{\exp }$ is the expected execution time of subtask $T_{i}$ and $T^{\exp }$ is the expected completion time of the total task).

$$
T C_{i}=\frac{T_{i}^{\exp }}{T^{\exp }} .
$$

Task coupling degree is the quantitative level of interaction between tasks. In the process of work collaboration, the interaction between tasks is mainly reflected in the interaction of information flow. If the coupling degree of subtasks is large, the interrelation between tasks is large and the dependence is strong, and the completion status of subtasks will have a great impact on other subtasks. The coupling degree of subtask $T_{i}$ is (where $T_{i}$ and $T_{j}$ are two independent subtasks, $\sum_{j=1}^{n} \operatorname{relate}\left(T_{i}, T_{j}\right)$ is the total amount of information interaction of task $T_{i}$ ):

$$
\mathrm{RC}_{i}=\left\{\begin{array}{l}
\sum_{j=1}^{n} \operatorname{relate}\left(T_{i}, T_{j}\right), \\
0, \quad n \leq 1 ;
\end{array}\right.
$$
follows:

Therefore, the importance degree of subtasks $\mathrm{IC}_{i}$ is as

$$
\mathrm{IC}_{i}=\alpha_{i 1} \mathrm{IC}_{i}+\alpha_{i 2} \mathrm{RC}_{i}
$$

where $\alpha_{i 1}+\alpha_{i 2}=1$, in which $\alpha_{i 1}$ and $\alpha_{i 2}$ are the proportion of the execution time and coupling degree of subtasks in the importance degree of tasks, respectively.

The complexity of subtask $T_{i} \mathrm{CC}_{i}$ is a description of the structure and function of the subtask, and it is also one of the important attributes that need to be considered when adjusting the scheme. The complexity of design tasks is related to the degree of integrated knowledge of tasks. The influence of the $n$ attributes on the complexity can be scored by experts, and the value range is $[0,1]$, and the evaluation matrix $\mathrm{ORI}_{n \times e}$ can be determined from this. According to the formula

$$
\operatorname{stan}_{i j}=\frac{\operatorname{ori}_{i j}}{\max _{i}\left(\operatorname{ori}_{i j}\right)},
$$

where $i$ is the number of experts and ori $i_{i j}$ is elements of the initial evaluation matrix, $\operatorname{stan}_{i j}$ is an element of the standardized evaluation matrix.

Subtasks with higher resource demand should be given priority as far as possible, where the demand intensity of subtask $T_{i}$ for a certain resource $\mathrm{DC}_{i, m}$ refers to the ratio of the demand degree of subtask for the resource to the total supply $s_{m}$ of the resource; that is,

$$
\mathrm{DC}_{i, m}=\frac{d_{i, m}}{s_{m}} .
$$

The total resource demand intensity of a subtask refers to the comprehensive demand intensity of the subtask for various resources, and the resource demand intensity DC of 
the subtask $T_{i}$ is calculated as follows (where $M$ is the total class of resources):

$$
\mathrm{DC}_{i}=\sum_{m=1}^{M} \frac{d_{i, m}}{s_{m}}
$$

If a task requires a large amount of resources, that is, the greater the value of resource demand intensity $\mathrm{DC}_{i}$, the greater the impact of the task on the working period, the task should give priority to matching resources and compete with other subtasks for resources in the process of product development, thus affecting the construction period. Therefore, the resource demand intensity coefficient of tasks should also be one of the indicators to measure whether tasks should be matched first.

Remember $\omega_{i}$ as the weight of index $x_{i}$, and the priority of subtask $T_{i}$ is as follows:

$$
\varphi(T)_{i}=\omega_{1 i} \omega I C_{i}+\omega_{2 i} \omega I C_{i}+\omega_{3 i} \omega I C_{i}+\cdots+\omega_{n i} \omega I C_{i},
$$

wherein $\omega_{1 i}, \omega_{2 i}, \ldots, \omega_{n i}$ denote the weight coefficients of task importance of task $T_{i}$, respectively, and $\omega_{1 i}+\omega_{2 i}+\omega_{3 i}+\ldots \omega_{n i}=1$.

According to the priority of tasks, the matching order of each subtask is sorted. The higher the priority, the priority matches the resources, and the lower the priority, the later matches the resources; at the same time, the higher the priority of the task, the greater the impact of the task delay on the whole process.

4.3. Construction of Optimization Model: Overview of Model. A city has several communities, each of which contains several departments, and each department contains several members. Tasks are distributed by superiors, which are divided into several subtasks through the integration and analysis of tasks. Subdepartments cooperate and combine into several teams $O_{j}$ through reasonable allocation of personnel. After resources are reasonably matched, the corresponding teams $O_{j}$ complete the corresponding subtasks $T_{i}$.

(1) Assigned tasks are related to various resources (based on time $t$ ): between combined teams and assigned tasks, between other updatable resources and assigned tasks, and between nonupdatable resources and assigned tasks.

(2) The execution time of assigned tasks after matching teams, other updatable resources, and nonupdatable resources is a nonnegative integer.

The expected completion time of assigned tasks is determined by the average level of resources based on past task execution experience.

(A) The establishment of the model is that the task satisfies various logical relations and constraints, which makes the total time of the task shortest, the total cost lowest, and the resource utilization most efficient.

(B) At any time $t$, a combined team and a single resource can only perform one assigned task.

(C) Before task matching begins, each free resource can be allocated freely.

\section{Hypothetical Case}

5.1. Optimal Matching of Public Management Resources in a City. A city has a total area of 82,400 square kilometers and has jurisdiction over 26 districts, 8 counties, and 4 autonomous counties. There are several communities, and each community contains several departments, and each department contains several members. Tasks are distributed by superiors, which are divided into several subtasks through the integration and analysis of tasks. Subdepartments cooperate and combine into several teams $O_{j}$ through reasonable allocation of personnel. After resources are reasonably matched, the corresponding teams $O_{j}$ complete the corresponding subtasks $T_{i}$.

\subsection{Work Task Priority Calculation}

5.2.1. Importance of the Task. According to the formula in this paper, the time importance of each task is calculated as shown in Table 1.

Based on the fuzzy evaluation method and the formula in this paper, the coupling degree of each task is calculated as shown in Table 2.

Assuming that the specific gravity coefficients of time importance and coupling are both 0.5 , based on Tables 1 and 2, calculate the importance of each task, and it can be obtained as shown in Table 3.

5.2.2. Complexity of the Task. According to the task complexity, experts evaluate and calculate the complexity of each task as shown in Table 4

5.2.3. Task Demand Intensity. According to the formula in this paper, the resource requirements of each task are calculated as shown in Table 5 .

According to the formula in this paper, the ability levels of each team in human resources can be calculated as shown in Figure 6.

According to the formula, the comprehensive preference information of each subtask of each team in human resources is calculated as shown in Figure 7.

According to the formula in this paper, the comprehensive matching degree of each team to subtasks in human resources can be calculated as shown in Figure 8 .

In Figures 6 to 8, when each task is executed on different goals, it can be seen that there are differences in some goals and tasks; for example, there are great differences in O_3 
Table 1: Time importance of each task.

\begin{tabular}{lcccccccc}
\hline Task number & $T_{1}$ & $T_{2}$ & $T_{3}$ & $T_{4}$ & $T_{5}$ & $T_{6}$ & $T_{7}$ & $T_{8}$ \\
\hline Time importance & 0.025 & 0.054 & 0.029 & 0.145 & 0.043 & 0.099 & 0.116 & 0.024 \\
\hline
\end{tabular}

TABle 2: Coupling degree of each task.

\begin{tabular}{lcccccccc}
\hline Task number & $T_{1}$ & $T_{2}$ & $T_{3}$ & $T_{4}$ & $T_{5}$ & $T_{6}$ & $T_{7}$ & $T_{8}$ \\
\hline Coupling degree & 0.0039 & 0.086 & 0.045 & 0.055 & 0.056 & 0.05 & 0.042 & 0.052 \\
\hline
\end{tabular}

TABLE 3: Importance of each task.

\begin{tabular}{lcccccccc}
\hline Task number & $T_{1}$ & $T_{2}$ & $T_{3}$ & $T_{4}$ & $T_{5}$ & $T_{6}$ & $T_{7}$ & $T_{8}$ \\
\hline Importance & 0.032 & 0.070 & 0.037 & 0.100 & 0.050 & 0.074 & 0.079 & 0.038 \\
\hline
\end{tabular}

TABle 4: Complexity of each task.

\begin{tabular}{lcccccccc}
\hline Task number & $T_{1}$ & $T_{2}$ & $T_{3}$ & $T_{4}$ & $T_{5}$ & $T_{6}$ & $T_{7}$ & $T_{8}$ \\
\hline Time importance & 0.006 & 0.053 & 0.009 & 0.168 & 0.030 & 0.147 & 0.137 & 0.019 \\
\hline
\end{tabular}

TABLE 5: Resource requirement information for each task.

\begin{tabular}{lccccc}
\hline Task number & \multicolumn{3}{c}{ Other renewable resources } & Nonupdatable resource & Resource requirements \\
\hline$T_{1}$ & 0.063 & 0.050 & 0.053 & 0.026 & 0.274 \\
$T_{2}$ & 0.125 & 0.050 & 0.105 & 0.053 & 0.489 \\
$T_{3}$ & 0.063 & 0.050 & 0.053 & 0.026 & 0.274 \\
$T_{4}$ & 0.063 & 0.050 & 0.105 & 0.026 & 0.364 \\
$T_{5}$ & 0.125 & 0.050 & 0.053 & 0.026 & 0.445 \\
$T_{6}$ & 0.125 & 0.050 & 0.105 & 0.053 & 0.398 \\
$T_{7}$ & 0.063 & 0.050 & 0.053 & 0.026 & 0.311 \\
$T_{8}$ & 0.063 & 0.100 & 0.105 & 0.026 & 0.396 \\
\hline
\end{tabular}

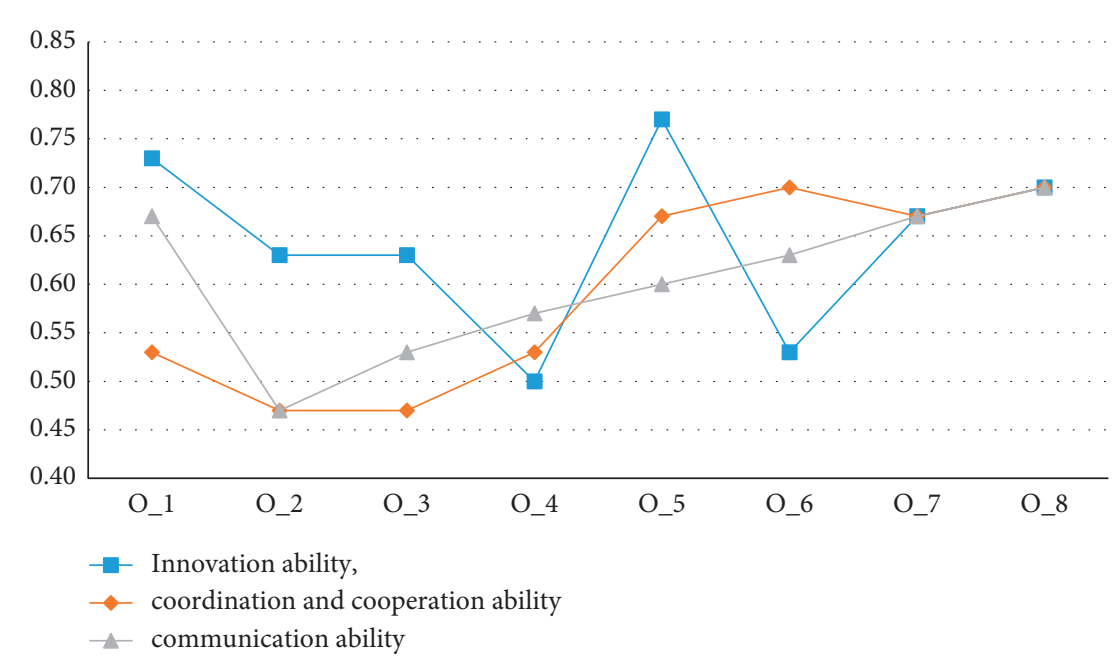

FIgURE 6: Competency levels for each team. 


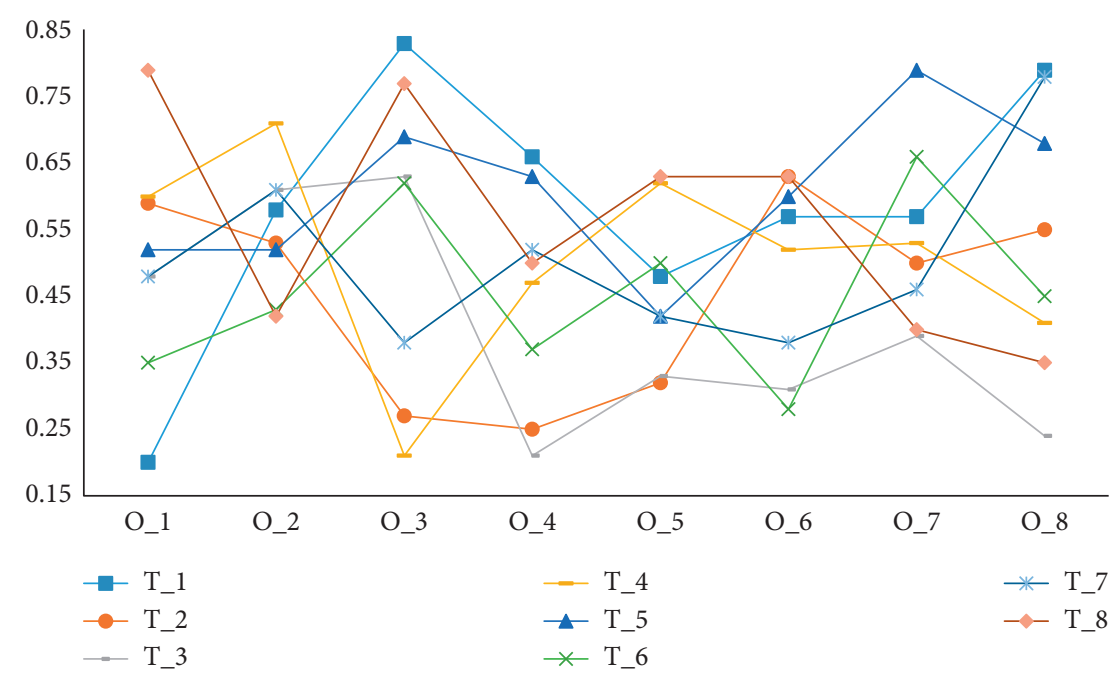

FIGURE 7: Comprehensive preference information of each subtask of each team.

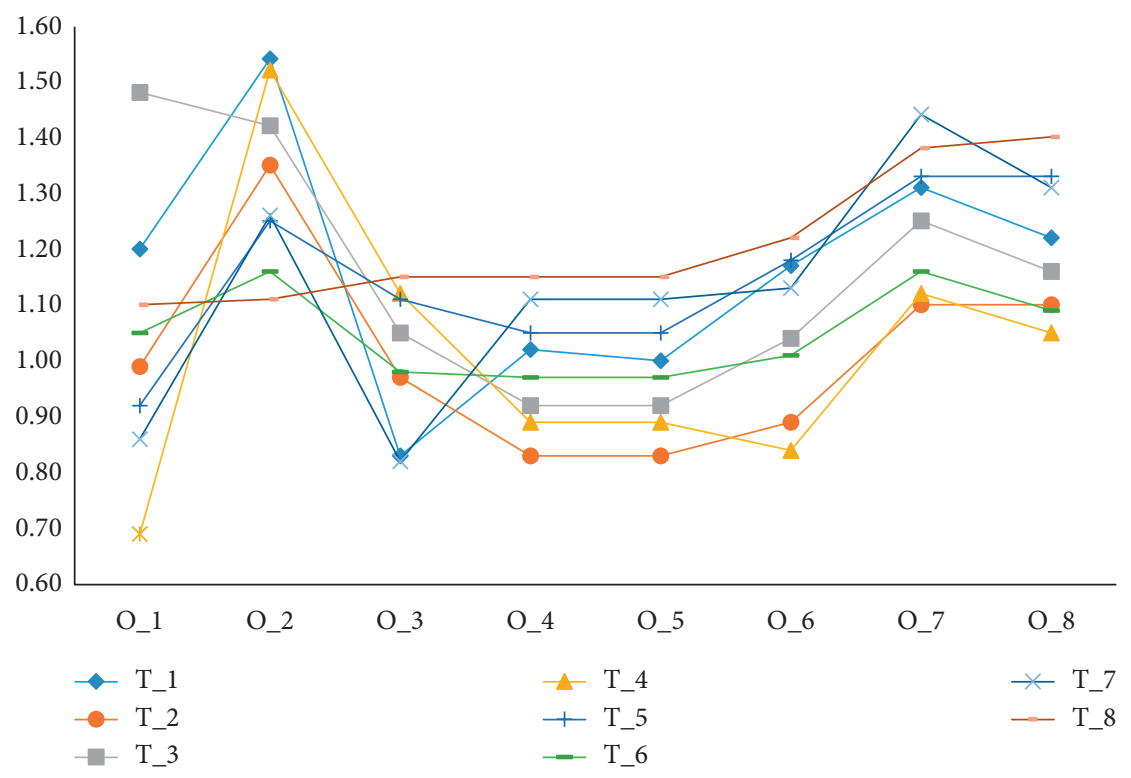

Figure 8: Comprehensive matching degree of each subtask of each team.

goals in Figure 7. However, some goals are consistent; for example, each goal in Figure 8 is highly homogeneous. Therefore, in the process of implementing each goal, the change of goal value in the task reflects the difference of each subtask.
Based on Table 5, the comprehensive resource requirements of each task can be obtained as shown in Table 6.

According to this paper, the weights of task importance, complexity, and resource demand are $0.4,0.4$, and 0.2 , respectively. The synthesis is shown in Table 7. 
TABLE 6: Comprehensive resource demand intensity of each task.

\begin{tabular}{lcccccccc}
\hline $\begin{array}{l}\text { Task } \\
\text { number }\end{array}$ & $T_{1}$ & $T_{2}$ & $T_{3}$ & $T_{4}$ & $T_{5}$ & $T_{6}$ & $T_{7}$ & $T_{8}$ \\
\hline $\begin{array}{l}\text { Demand } \\
\text { intensity }\end{array}$ & 0.038 & 0.069 & 0.038 & 0.051 & 0.062 & 0054 & 0.043 & 0.055 \\
\hline
\end{tabular}

Table 7: Priority of each task.

\begin{tabular}{lcccccccc}
\hline $\begin{array}{l}\text { Task } \\
\text { number }\end{array}$ & $T_{1}$ & $T_{2}$ & $T_{3}$ & $T_{4}$ & $T_{5}$ & $T_{6}$ & $T_{7}$ & $T_{8}$ \\
\hline $\begin{array}{l}\text { Demand } \\
\text { intensity }\end{array}$ & 0.023 & 0.063 & 0.026 & 0.117 & 0.044 & 0.099 & 0.095 & 0.034 \\
\hline
\end{tabular}

\section{Conclusion}

This paper analyzes the matching of two categories of public management resources: renewable resources and nonrenewable resources, and then studies and analyzes the priority of subtasks in the total task to calculate and judge. Therefore, the application of multiobjective optimization in intelligent algorithm can effectively solve the optimization matching problem of public management resources. Although this algorithm has achieved some results, there are still some problems in practical application, and the next step will be an in-depth study to find a better matching.

\section{Data Availability}

The experimental data used to support the findings of this study are available from the corresponding author upon request.

\section{Conflicts of Interest}

The authors declared that they have no conflicts of interest regarding this work.

\section{Acknowledgments}

This work was sponsored in part by the National Social Science Foundation of China (grant no. 14BG071).

\section{References}

[1] J. Wang, Li Chao, X. Zhang et al., "Data-centric smart city research review," Computer Research and Development, vol. 51, no. 2, pp. 239-259, 2014.

[2] F. Santoso and S. J. Redmond, "Indoor location-aware medical systems for smart homecare and telehealth monitoring: stateof-the-art," Physiological Measurement, vol. 36, no. 10, pp. R53-R87, 2015.

[3] L. Bo, R. Greevy, X. Xu et al., "Optimal nonbipartite matching and its statistical applications," The American Statistician, vol. 65, no. 1, pp. 21-30, 2011.

[4] A. Tnase and G. Popa, "Innovation of public administration through new human resources techniques and employee motivation," in Proceedings of the International Management Conference. Faculty of Management, Academy of Economic Studies, Bucharest, Romania, November 2019.
[5] R. Arbolino, R. Boffardi, L. De Simone, and G. Ioppolo, "Multi-objective optimization technique: a novel approach in tourism sustainability planning," Journal of Environmental Management, vol. 285, no. 4, Article ID 112016, 2021.

[6] Hergüner, "Preparing future public administrators: a study on the factors affecting cultural competence of public administration students," Evaluation and Program Planning, vol. 86, Article ID 101914, 2021.

[7] D. Liu, Z. Yao, and L. Chen, "Emergency scheduling optimization simulation of cloud computing platform network public resources," Complexity, vol. 2021, Article ID 9950198, 11 pages, 2021.

[8] W. Wang, L. Meng, L. Wu, and J. Hu, "Research on the main problems and countermeasures in the construction of university library informatization," IOP Conference Series: Materials Science and Engineering, vol. 799, Article ID 012027, 2020.

[9] N. James, "Logical, critical and creative: teaching 'thinking skills' to law students," Queensland University of Technology Law \& Justice Journal, vol. 12, no. 1, pp. 66-88, 2011.

[10] L. I. Hong, "Current situation. Problems and optimization countermeasures of rice and shrimp cultivation industry in Hubei Province," Asian Agricultural Research, vol. 12, no. 05, pp. 15-17+21, 2020.

[11] C. Liu, X. Wang, and Y. He, "Research on manufacturing resource modeling based on the O-O method," Journal of Materials Processing Technology, vol. 139, no. 1-3, pp. 40-43, 2003.

[12] K. Deb, A. Pratap, S. Agarwal, and T. Meyarivan, "A fast and elitist multiobjective genetic algorithm: NSGA-II," IEEE Transactions on Evolutionary Computation, vol. 6, no. 2, pp. 182-197, 2002.

[13] C. C. Coello and M. S. Lechuga, "MOPSO:A proposal for multiple objective particle swarm optimization," IEEE Computer Society, vol. 2, pp. 1051-1056, 2002.

[14] F. Tao, Y. Cheng, L. Zhang, and D. Zhao, "Utility modelling, equilibrium, and coordination of resource service transaction in service-oriented manufacturing system," Proceedings of the Institution of Mechanical Engineers-Part B: Journal of Engineering Manufacture, vol. 226, no. 6, pp. 1099-1117, 2012.

[15] M. Sheikhan and Z. Jadidi, "Flow-based anomaly detection in high-speed links using modified GSA-optimized neural network," Neural Computing \& Applications, vol. 24, no. 3, pp. 599-611, 2014. 